\title{
The Expression and Relationship of CD68-Tumor- Associated Macrophages and Microvascular Density With the Prognosis of Patients With Laryngeal Squamous Cell Carcinoma
}

\author{
Shujun Sun ${ }^{1, \star} \cdot$ Xinliang Pan ${ }^{1} \cdot$ Limin Zhao ${ }^{2} \cdot$ Jianming Zhou ${ }^{2} \cdot$ Hongzeng Wang $^{2} \cdot$ Yonghong Sun ${ }^{3}$ \\ ${ }^{1}$ Department of Otorhinolaryngology, Qilu Hospital of Shandong University, Jinan; Departments of ${ }^{2}$ Otorhinolaryngology and ${ }^{3}$ Pathology, \\ The Affiliated Hospital of Weifang Medical University, Weifang, China
}

Objectives. We sought to identify the expression of CD68-tumor-associated macrophages (TAMs) and CD34-microvascular density (MVD) in laryngeal squamous cell carcinoma (LSCC), to study the relationship with clinical pathological parameters and to determine whether their expression is predictive of disease.

Methods. Pathologically confirmed 45 LSCC tissue and 20 peritumoral non-tumor tissue were examined. Immunohistochemical studies were used to detect the expression of CD68-TAMs and CD34-MVD.

Results. The positive expression rate of CD68 in LSCC tissue was 82\% (37/45), which was higher than the 10\% (2/20) expression rate of the peritumoral tissue $(P<0.05)$. The CD34-MVD positive expression rate in the LSCC tissue was $26.5 \pm 6.4$, which obviously higher than $12.2 \pm 4.0$ expression rate of the peritumoral tissue $(P<0.05)$. The positive expression rates of both CD68 and CD34-MVD were higher in the lymph node metastasis (LNM) positive group than in the LNM negative group. The expression of CD68 had positive correlation with CD34-MVD. The 5-year disease-free survival rate in the group with the low CD68 expression was significantly higher than that in the group with high CD68 expression (76\% vs. $42 \%$, respectively).

Conclusion. The high expression of CD68-TAMs in LSCC and its positive correlation with CD34-MVD illustrates that both play an important role in promoting the metastasis and angiogenesis of this cancer. Their expression was also positively correlated with the prognoses of these patients, suggesting that they could be used as important prognostic markers for LSCC.

Keywords. Laryngeal Neoplasms; Tumor-Associated Macrophages; CD; Microvascular Density

\section{INTRODUCTION}

- Received August 21, 2015

Revised December 5, 2015

Accepted January 12, 2016

- Corresponding author: Xinliang Pan

Department of Otorhinolaryngology, Qilu Hospital of Shandong University, Jinan 250012, China

Tel: +86-531-82169570, Fax: +86-531-82169570

E-mail: XinLiangPancn@126.com

*The first author is now working at the Department of Otolaryngology, the Affiliated Hospital of Weifang Medical University, Weifang, China.
Despite recent improvements in surgery and radiation therapy, treatment failures still occur in laryngeal squamous cell carcinoma (LSCC) patients. Therefore, the development of novel and effective therapeutic strategies and an increased understanding of the relevant biomarkers and their effects on therapeutic responses may improve LSCC patient prognoses.

Cancer-associated fibroblasts are the most prominent cell type within the tumor stroma of many cancers and an important player in the tumor microenvironment which consists of a dynamic mixture of fibroblasts, monocytes/macrophages, endothe-

Copyright (C) 2016 by Korean Society of Otorhinolaryngology-Head and Neck Surgery.

This is an open-access article distributed under the terms of the Creative Commons Attribution Non-Commercial License (http://creativecommons.org/licenses/by-nc/4.0)

which permits unrestricted non-commercial use, distribution, and reproduction in any medium, provided the original work is properly cited. 
lial cells, lymphocytes, and granulocytes [1]. These noncancerous cells provide many paracrine signals and necessary to turn on the pleotrophic abilities of cancer cells [2]. For example, fibroblasts can become cancer-associated fibroblasts and macrophages [3].

Macrophages that infiltrate tumor tissues are referred to as 'tumor-associated macrophages' (TAMs) and closely involved in tumorigenesis by inducing angiogenesis, immunosuppression, and invasion. TAMs are present with varying frequency in tumors, and are common in head and neck squamous cell carcinomas [4]. Accordingly, studies in various cancers have shown that TAMs can be associated with both a positive or negative prognosis [5]. TAMs express several macrophage-specific markers including CD68, CD23, and CD14. They also express the vascular endothelial growth factors (VEGF) $\mathrm{C}$ and $\mathrm{D}$ and the specific tyrosine kinase receptorVEGF receptor-3.

Whilst TAMs are generally oriented towards promoting tumor growth and angiogenesis and suppressing adaptive immunity. They also have an important role in tumor cell migration, invasion, and metastasis [6]. Few studies have investigated microenvironment of head and neck cancer on tumor progression [7]. Several reports have suggested that TAMs are associated with tumor growth, disease progression, and poor prognosis in some human cancers $[8,9]$. Recent studies from our group demonstrated an overexpression of CD68-TAMs in LSCC. More importantly, we identified TAMs as one of the tumor microenvironment in the tumor-macrophage interaction and showed that laryngeal cancer cells released TAMs, which, subsequently, promote tumor migration. There are fewer reports about the relationship between CD68-TAMs and microvascular density (MVD) [10]. Despite progress in understanding the interplay between inflammation and cancer, important questions remain unanswered. It is not known if the survival rate of laryngeal cancer patients is affected by CD68-TAMs and MVD after surgery, or if these could be important prognostic markers of laryngeal cancer.

\section{H I G H L I G H T S}

- CD68-positive tumor-associated macrophages were expressed in 37 of 45 laryngeal cancer samples.

- CD34-positive microvascular density were expressed in 2 of 20 peritumoral non-tumor tissue samples.

- Lymph node metastasis and lymph node metastasis assist stage were risk factors for laryngeal squamous cell carcinoma $(P<0.05)$.

- The CD68 and CD34 positivity were significantly associated with lymph node metastasis.

- High CD68 expression in laryngeal tumors was associated with poor 5-year disease-free survival.
In the present study, we explored the expression of CD68TAMs and CD34-MVD in LSCC tissue and their relationship with clinicopathological parameters, such as survival rates, in order to identify any potential theoretical basis for using these molecular markers for clinical guide and intervention.

\section{MATERIALS AND METHODS}

\section{Patient characteristics}

A total of 45 patients with histologically confirmed laryngeal carcinoma, who underwent surgical treatment in the Department of Otolaryngology (the Affiliated Hospital of Weifang Medical University) between August 2008 and October 2014 were included in this study. All cases were diagnosed with squamous cell carcinoma and confirmed by postoperative pathological examination. As a control group, adjacent non-tumor normal tissues were obtained from 20 cases. These control samples were contralateral hemi-laryngeal normal tissue or mucosal tissue more than 1.5-cm distance from the LSCC tumor. None of the 45 patients received preoperative chemotherapy and/or radiotherapy. This study was conducted in accordance with the declaration of Helsinki. This study was conducted with approval from the Ethics Committee of Shandong University (KYLL-2014 (KS)-192). Written informed consent was obtained from all participants.

We recorded the age of the patient, diagnosis, clinical stage, the discharge time, postoperative radiotherapy and/or chemotherapy, the location, pathological type, and differentiation degree. The time of death, the time and site of recurrence, and the time for loss of visitor were identified by telephone follow-up and from outpatient care records.

\section{Sample preparation}

Specimens were fixed with $10 \%$ formalin after surgical removal and $5-\mu \mathrm{m}$ continuous sections were prepared by routine pathological examination. All sections were dried at $42^{\circ} \mathrm{C}$ for 6 hours and deparaffinized. For each sample, one section was prepared for routine hematoxylin-eosin staining and others for immunohistochemical staining.

\section{Immunohistochemical staining}

The sections were heated in trisodium citrate buffer at $100^{\circ} \mathrm{C}$ for 20 minutes for antigen retrieval. The sections were incubated with CD68 antibody and CD34 antibody for 2 hours at room temperature. Mouse anti-human CD68 monoclonal antibody (1:100) and mouse anti-human CD34 monoclonal antibody were purchased from Beijing Sequoia Jinqiao Biological Technology Co. (Beijing, China). Then, the sections were incubated with Envision second antibody at room temperature for $30 \mathrm{~min}$ utes. Diaminobenzadine was used to reveal specific binding, followed by hematoxylin counterstaining, dehydration, clearing, 
and neutral gum sealing. Phosphate buffer saline (PBS) in place of the primary antibody served as the control.

Under a light microscope, the clear background, positive expression in the cytoplasm were brown-yellow particles. Peroxidase blocking buffer, $0.01 \mathrm{~mol} / \mathrm{L}(\mathrm{pH} 6.0)$ trisodiumcitrate antigen retrieval solution, and $0.01 \mathrm{~mol} / \mathrm{L}$ (pH 7.4) PBS were purchased from Maxim Biotech Co. (San Francisco, CA, USA). The positive expression of CD68 was identified by brownish-yellow stained granules in the tumor cell cytoplasm and there was less perinuclear expression in the stroma. CD34-MVD mainly expressed in endothelial cell plasma, characterized by diffuse distribution because of many vessels in the interstitial cells. More than $10 \%$ cellular staining was defined as a positive reaction and $<10 \%$ was considered as a negative reaction. Low expression level (+) was defined as $10 \%$ to $25 \%$ cell were positive; moderate expression level (++) was deficned as $26 \%$ to $50 \%$ cells were positive; and strong expression level (+++) was defined as $>50 \%$ cells were positive.

The positive expression of CD68-TAMs in the cytoplasm of macrophages appeared as yellowish-brown to brown granules. Two pathologists calculated the positive expression of CD68, as an expression of the infiltration of LSCC by CD68-TAMs, using the average value of five high magnification visual fields. The average value of all specimens was counted, and judgment standard of positive expression in benign lesions the same as that of above malignant lesions.

CD34 antibody staining showed the MVD of tumors. In circumstances of unknown clinical and pathological features, two pathologists screened out the highest MVD region at low magnification (at the tumor/stromal edge or tumor interstitial microvessel density). The pathologists then recorded five highpower (200 times magnification) fields of view and estimated the average tumor microvessel count.

\section{Statistical analysis}

The results were analyzed with $t$-tests, chi-square tests, and Spearman rank method after a homogeneity test for variance. All data were analyzed with SPSS ver. 13.0 (SPSS Inc., Chicago, IL, USA), using $P<0.05$ as the detection standard. Cases were divided into groups according to their TAMs density and these groups were analyzed by the Kaplan-Meier method. We analyzed prognostic multi-factors using the Cox regression model.

\section{RESULTS}

\section{Selection of clinical cases}

A total of 45 patients were confirmed by biopsy to have LSCC prior to surgery. According to the tumor-node-metastasis (TNM) classification standard published by the Union for International Cancer Control in June, 2002, there were 15 patients with stage $\mathrm{T} 1,17$ patients with stage T2, 9 patients with stage T3, and 4 pa- tients with stage T4. Twenty-eight tumors were in the supraglottic area and 17 tumors were in the glottic area; and there were 26 cases with cervical lymph node metastases and 19 cases without lymph node metastases. The number of well, moderately, and poorly differentiated tumors was 20,18 , and 7 , respectively. The age of the patients ranged from 43 to 76 years old, and the median patient age was 65.3 years old. There were 18 males and 2 females as control group with adjacent non-tumor normal tissues.

\section{Expression of CD68-TAMs and CD34-MVD}

The positive expression of CD68 was brownish-yellow granules in the cytoplasm and perinuclear area of peritumoral stroma and tumor tissues in laryngeal squamous cell carcinoma (Fig. $1 \mathrm{~A}, \mathrm{~B})$. There is little or no expression in adjacent non-tumor normal tissues (Fig. 1C). The positive expression rate of CD68 was $82 \%(37 / 45)$ in tumor tissues and $10 \%(2 / 20)$ in adjacent non-tumor tissues $(P<0.05)$. The degree of CD68 positive expression was moderate and strong positive to see more, infiltrating macrophages were positive expression around the tumor.

According to the expression of CD68, we selected five fields of view at high magnification and counted the number of positive cells. The range was 1-133/high power field (HP), with a mean of $62.7 / \mathrm{HP}$ and a median of 65.3/HP. For the infiltration and expression of $\mathrm{CD} 68$, we divided the cases at the median into a high CD68 expression (high infiltration area) group $(\mathrm{n}=24)$ and a low CD68 expression (low infiltration area) group $(\mathrm{n}=21)$.

The expression of CD34-MVD focused on microvascular endothelial cells in the tumor nest's edge zone with diffuse distribution. The cancer center has necrotic tissue and few new blood vessels, so CD34-MVD expression is lower in this zone (Fig. 1D, E). The average microvascular count in 5 high magnification fields of view in tumor tissues was $26.5 \pm 6.4$ and in adjacent non-tumor normal tissues was $12.2 \pm 4.0(P<0.05)$.

\section{Correlation of survival rate and CD68 expression}

During a median follow-up of 48.3 months (range, 3 to 60 months), 7 patients (16\%) had died of their disease, 3 patients $(7 \%)$ had died of other causes, and there were 35 survival patients $(78 \%)$ in the follow-up time. In the CD68 low expressing cases, the 2-year locoregional control (LRC), disease-free survival (DFS), and overall survival (OS) were $86 \%, 80 \%$, and $79 \%$, respectively, and the 5 -year LRC, DFS, and OS were $68 \%$, $76 \%$, and $66 \%$, respectively. In the CD68 high expressing cases, the 2 -year LRC, DFS, and OS was $71 \%, 69 \%$, and $67 \%$, respectively, and the 5 -year LRC, DFS, and OS was $38 \%, 42 \%$, and $35 \%$, respectively.

In the 21 CD68 low expressing cases, the average survival time was $106.1 \pm 10.2$ months and the 5 -year survival rate was $76 \%(16 / 21)$. In the 24 CD68 high expressing cases, the average survival time was $56 \pm 9.1$ months and the 5 -year survival rate 

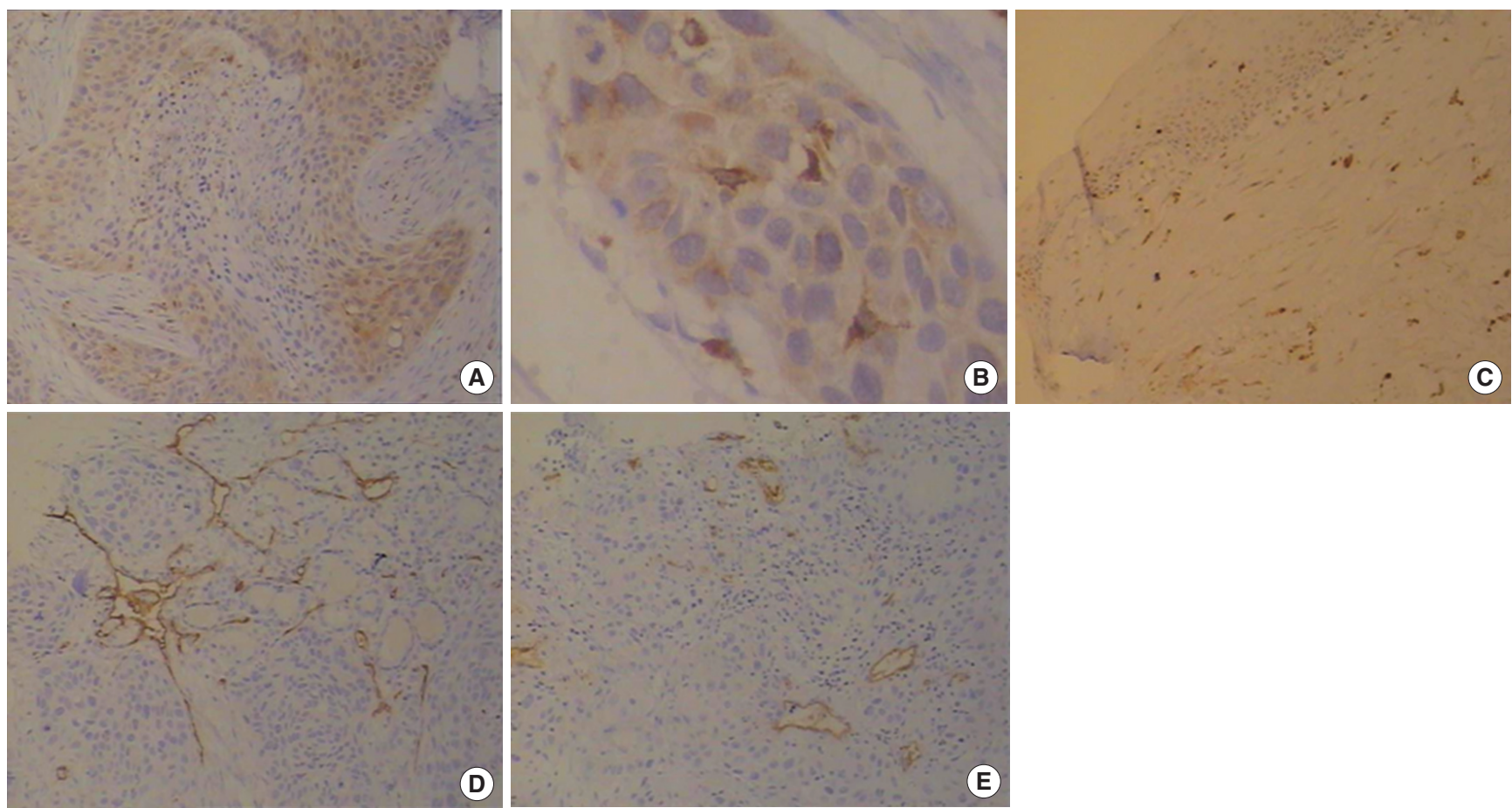

Fig. 1. (A) Expression of CD68 in laryngeal squamous cell carcinoma SP (×200). (B) Expression of CD68 in laryngeal squamous cell carcinoma SP $(\times 400)$. (C) Little or no expression in adjacent non-caner normal tissues SP (X200). (D) Expression of CD34 in tumor nests edge zone SP (X200). (E) Expression of CD34 in Laryngeal squamous cell carcinoma of the inside edge of tumor tissue stroma SP (X200). SP, streptavidin-perosidase.

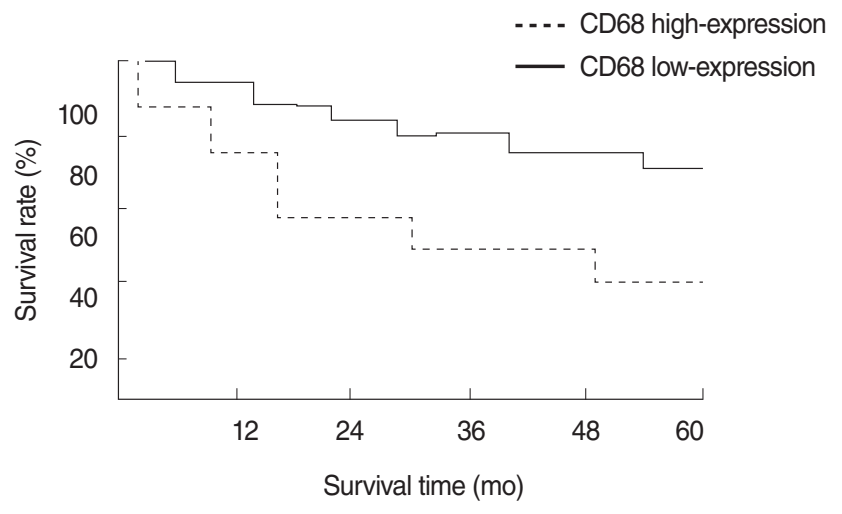

Fig. 2. The survival curves in the low infiltration area (low expression zone) and high infiltration area (highly expression area) of CD68 in laryngeal squamous carcinoma. The average survival time was 106.1 10.2 months in CD68 low-expression area, 5-year survival rate was $76 \%$ (16/21). The average survival time was $56.3 \pm 9.1$ months in the high-expression area, 5-year survival rate was $42 \%$ (10/24). There was statistical significance between two by log-rank method $(P=0.020)$.

was $42 \%$ (10/24) (log-rank method, $P=0.020)$ (Fig. 2).

Relationships between CD68-TAMs, CD34-MVD, and clinicopathological parameters

The CD68 positive expression rate in the glottis and supraglottic areas was $71 \%(12 / 17)$ and $89 \%(25 / 28)$, respectively $\left(\chi^{2}=2.54\right.$, $P>0.05)$, suggesting that the infiltration of CD68-TAMs has no correlation with the location of tumor growth. The CD68 positive expression rate in the LNM positive and LNM negative groups was $100 \%(26 / 26)$ and $58 \%(11 / 19$, respectively; $P<0.05)$. CD68 positive expression had no correlation with the degree of tumor differentiation, but correlated with clinical stage.

CD34-MVD expression was significantly higher in the LNM positive group than the LNM negative group $(P<0.05)$. There was no significant difference in the tumor location, the degree of differentiation, or the TNM staging $(P>0.05)$. The high expression of CD34-MVD in LNM positive tumors is likely because the tumor has new, stronger blood vessels forming and increased metastatic activity and ability (Table 1).

\section{Univariated and multivariated analyses for recurrence and survival}

We analyzed the expression of CD68, CD34-MVD, age, clinical stage, size of postoperative residual lesion, histological grade, postoperative radiotherapy, and pathological types by the Cox regression model, the results show that the infiltration degree of CD68-TAMs, the expression of CD34-MVD, and the histological grading were prognosis factors for patients with laryngeal cancer. Indeed, the relative risk for a CD68-TAMs infiltration density was greater than 2 , indicating that the higher CD68TAMs infiltrating density, the worse the prognosis (Table 2). 
Table 1. The relationship between expression of CD68, CD34-MVD, and clinical pathological parameters $(\bar{\chi} \pm s)$

\begin{tabular}{|c|c|c|c|c|c|c|c|c|}
\hline \multirow{2}{*}{ Parameter } & \multirow{2}{*}{ Case } & \multicolumn{2}{|c|}{ CD68 } & \multirow{2}{*}{$\chi^{2}$} & \multirow{2}{*}{$P$-value } & \multirow{2}{*}{ MVD } & \multirow{2}{*}{$T$-value } & \multirow{2}{*}{$P$-value } \\
\hline & & - & + & & & & & \\
\hline Age (yr) & & & & & $>0.05$ & & & $>0.05$ \\
\hline$<60$ & 13 & 3 & 10 & 0.79 & & $24.2 \pm 4.2$ & 1.74 & \\
\hline$\geq 60$ & 32 & 4 & 28 & & & $27.2 \pm 3.6$ & & \\
\hline Location & & & & & $>0.05$ & & & $>0.05$ \\
\hline Glottic & 17 & 5 & 12 & 2.54 & & $22.1 \pm 4.6$ & 1.92 & \\
\hline Supraglottic & 28 & 3 & 25 & & & $28.5 \pm 3.2$ & & \\
\hline Lymph node metastasis & & & & & $<0.05$ & & & $<0.05$ \\
\hline Yes & 26 & 0 & 26 & 13.30 & & $31.5 \pm 6.6$ & 2.86 & \\
\hline No & 19 & 8 & 11 & & & $21.7 \pm 6.8$ & & \\
\hline Degree of differentiation & & & & & $>0.05$ & & & $>0.05$ \\
\hline High & 20 & 6 & 14 & 3.71 & & $25.5 \pm 6.5$ & 1.81 & \\
\hline Moderate & 18 & 2 & 16 & & & $27.5 \pm 6.3$ & & \\
\hline Low & 7 & 0 & 7 & & & $28.2 \pm 7.2$ & & \\
\hline Tumor-node-metastasis stage & & & & & $<0.05$ & & & $>0.05$ \\
\hline$|-| \mid$ & 32 & 8 & 24 & 13.12 & & $25.6 \pm 5.6$ & 1.33 & \\
\hline III-IV & 13 & 0 & 13 & & & $28.2 \pm 7.7$ & & \\
\hline
\end{tabular}

MVD, microvascular density.

Table 2. Multiple factors analysis for the prognosis of patients with laryngeal cancer

\begin{tabular}{lcc}
\hline Factor & $P$-value & Relative risk \\
\hline $\begin{array}{l}\text { Infiltration degree of CD68-tumor- } \\
\quad \text { associated macrophages }\end{array}$ & $<0.001$ & 3.33 \\
$\begin{array}{l}\text { Expression of CD34-microvascular } \\
\quad \text { density }\end{array}$ & $<0.003$ & 1.85 \\
Histological grade & $<0.004$ & 1.53 \\
Age & 0.771 & 0.25 \\
Clinical stage & 0.025 & 0.98 \\
Pathological types & 0.019 & 0.92 \\
Postoperative residual lesion size & 0.017 & 0.89 \\
Postoperative radiotherapy & 0.032 & 0.79 \\
\hline
\end{tabular}

\section{The correlation of CD68 expression and CD34-MVD}

The blood counts of CD34-MVD were $28.7 \pm 6.2$ and $16.3 \pm 4.1$ in the CD68 positive group and negative group, respectively $(t=2.51, P<0.05)$. The average CD34-MVD was $18.6 \pm 4.4$ in CD68 positive cases $(n=37)$ and $16.3 \pm 4.1$ in CD68 negative cases $(n=8)$. This result suggests that the infiltration of CD68TAMs and angiogenesis were positively correlated, and the higher CD34-MVD count showed the higher CD68 positive rate $(r=0.31, P<0.05)($ Table 3$)$.

\section{DISCUSSION}

In this study, we first examined the expression pattern and clinical significance of CD68-TAMs and CD34-MVD in LSCC. Although histological categories and tumor location was not correlated with the expression of CD68-TAMs and CD34-MVD,
Table 3. Relationship of expression of CD68 with CD34-MVD ( $\bar{\chi} \pm s)$

\begin{tabular}{lcccc}
\hline \multirow{2}{*}{ Variable } & \multirow{3}{*}{ Case } & \multicolumn{3}{c}{ CD68 } \\
\cline { 3 - 5 } & & Positive & Negative & Positive rate \\
\hline CD68 & 45 & $37^{\text {a) }}$ & 8 & 82.2 \\
CD34-MVD & & $28.7 \pm 4.2^{\text {b) }}$ & $16.3 \pm 4.1^{\text {c) }}$ & $18.6 \pm 4.4$ \\
\hline
\end{tabular}

There was significant difference between ${ }^{b)}$ and ${ }^{c)}$ negative group $(P<0.05)$. There was positively correlated with ${ }^{\text {b) }}$ and ${ }^{\text {a) }}$ the positive expression of CD68-tumor-associated macrophages $(r=0.31, P<0.05)$.

MVD, microvascular density.

clinical stage and lymph node metastases were associated with CD68-TAMs and therefore it could be defined as an important prognostic factor in LSCC. Lin et al. [11] found that infiltration of TAMs in supraglottic laryngeal carcinoma can be used to predict metastases and prognosis and is an independent factor for prognosis.

We also found that there was an increased expression of CD68-TAMs near the necrotic regions of tumors. The positive expression rate of CD68 in tumor tissues was $82 \%(37 / 45)$ and in tumor adjacent non-tumor tissues was $10 \%(2 / 20)(P<0.05)$. Maybe the necrotic regions of tumors have increased hypoxic conditions, prompting TAMs to play a greater role. Klimp et al. [12] found that hypoxia is an important condition for the vascular regeneration and activation of TAMs. In hypoxia and the stimulation of the tumor microenvironment, macrophages upregulate the expression of VEGF, fibroblast growth factor, thymidine phosphorylase, urokinase plasminogen activator, tumor necrosis factor- $\alpha$, COX-2, and other angiogenic factors.

TAMs play a role in the growth and invasion of tumor by hypoxia [13]. We found that the positive expression rate of CD68 in our 45 LSCC cases was $82 \%$, which was far higher than the 
$10 \%$ positive expression rate in the 20 adjacent non-tumor tissues. Expression of CD68 had no relationship with tumor site, but the positive expression of CD68 in the central tumor region was lower than around the tumor region, this situation was related with the necrotic tissues in the central region of the tumor. Marginal macrophage density, but not intratumoral macrophage density, was associated with vascular invasion, tumor multiplicity, and fibrous capsule formation. Our results demonstrate that high macrophage infiltration predicts poor prognosis in patients with LSCC. These results, together with our previous report showing the distinct activation patterns of macrophages in different areas of tumor tissue, implies that macrophages in those areas may use different strategies to promote tumor progression [14].

From this point of view, we selected segment tumor tissues in laryngeal carcinoma specimens, comparing the central area and the marginal area for the expression of CD68. There was no difference between central area and marginal area of the tumor, but the degree of positive expression of CD68 in the marginal area was higher than the center area, this situation may exist because there is a lot of inflammation and neo-vascularization in the marginal area of the tumor. Green et al. [15] revealed colony-stimulating factor (CSF)-1 as the major chemoattractant for macrophages, suggesting that these cells are drawn to actively invading areas of the tumor, but not the hypoxic core of the tumor mass. Their findings suggest a model where normoxic tumor cells release CSF-1 to recruit macrophages to the tumor periphery where they then secrete motility and angiogenic factors that facilitate tumor cell invasion and metastasis.

The tumor microenvironment is a complex ecology of cells that evolves with and provides support to tumor cells during the transition to malignancy. Among the innate and adaptive immune cells recruited to the tumor site, macrophagesare particularly abundant and are present at all stages of tumor progression [16]. The tumor stroma has long been ignored as a potential therapeutic target, but it has become clear that several stromal cell types play a non-redundant role during tumor progression. In particular, macrophages possess the capacity to stimulate tumor growth and metastasis via multiple mechanisms [17]. Clinical studies and experimental mouse models indicate that these macrophages generally play a protumoral role. In the primary tumor, macrophages can stimulate angiogenesis and enhance tumor cell invasion, motility, and intravasation. During monocytes and/or metastasis, macrophages prime the premetastatic site and promote tumor cell extravasation, survival, and persistent growth $[18,19]$.

Clinical studies have also demonstrated a close association between infiltration of TAMs and poor prognosis in patients with various human malignancies [20], suggesting that elevated inflammatory responses in the tumor microenvironment are important for malignant progression. Jung et al. [21] investigated TAM density in human tissue microarray sections from 14 different types of human cancers and normal thyroid, lung, and breast tissues. They concluded that cancers with higher TAM densities (pancreas, lung, anaplastic thyroid, and gallbladder) were associated with poor survival rate.

We considered the clinical function of TAMs and their relationship with other factors, and we selected MVD to examine whether a causal link exists. We found the expression of CD34MVD focused on microvascularendothelial cells in the tumor nest's edge zone, which showed diffuse distribution. However, the cancer center zone has necrotic tissue and fewer new blood vessels, so CD34-MVD had reduced expression in this zone. The average microvascular count in 5 high magnification fields of view for the tumor was $26.5 \pm 6.4$ and for the adjacent non-tumor normal tissues was $12.2 \pm 4.0(P<0.05)$.

Tumor vascularization is influenced by many molecular and cellular events in the tumor microenvironment, since transformed cells secrete pro-angiogenic molecules that recruit and activate not only endothelial cells, but also stromal cells such as macrophages [22]. Accumulating evidence suggests that TAMs act as key effectors, provoking a pro-angiogenic outcome during the 'angiogenic switch,' and play a prominent role in stimulating tumor angiogenesis and progression [23]. Moreover, we found an increased expression of CD34-MVD in tumor stromal cells, although the positive expression of CD34-MVD was in the microvascular endothelial cells of LSCC. CD34-MSD expression distribution was diffuse; however, there was more expression in the tumor nest's edge region, and there was little expression in the tumor central region. The high expression of macrophages suggests a mechanism by which tumor growth, angiogenesis, lymphangiogenesis, and lymph node metastasis are enhanced in highly metastatic cancer cells. TAMs are characterized by high expression of immunosuppressive and pro-angiogenic molecules, such as interleukin-10 and VEGF-A [24]. Similar results for lymphangiogenesis, angiogenesis, and infiltration of M2 macrophages expressing VEGF-A and VEGF-C were obtained in the syngeneic mouse model [25].

The growth and invasion of a tumor relies on the formation of new blood vessels and MVD can reflect the angiogenesis density. In some human tumors, TAMs promotes angiogenesis and expression of VEGF and PDGF. Angiogenesis plays an important role in tumor growth, metastasis, and prognosis. Bolat et al. [26] found that VEGF expression was correlated with high vascularityin infiltrative ductal carcinoma (IDC). TAMs contribute to tumor proliferation, progression, and angiogenesis, and have a complex role in tumor biology. Bolat et al. [26] found that MVD showed a significant positive correlation with TAMs, VEGF, metastatic lymph nodes, tumor size, and tumor grade in IDC. We found that the degree of infiltration of CD68-TAMs was positively correlated with angiogenesis-the higher CD34MVD count, the higher CD68 positive rate (Table 3).

High numbers of intratumoral TAMs are often correlated with poor prognosis, and recent studies have also highlighted that an increased presence of macrophages correlates with tumor me- 
tastasis to distant organs. Many authors have studied the relationship of TAMs and the prognosis of patients with malignant tumor and come to a similar conclusion as us. Zhang et al. [27] found that ovarian cancer patients with increased overall or intraislet M1/M2 TAM ratios presented with an improved 5-year prognosis. Nevertheless, the TAM distribution patterns did not influence the overall outcomes of the different ovarian cancer histotypes. Lin et al. [28] found that osteopontin (OPN) can skew macrophages toward M2TAMs during gastric cancer progression. The co-existence of OPN and infiltrating M2TAMs correlates with disease progression and poor survival, and thus can serve as a prognostic marker in gastric cancer. Chen et al. [29] found M2 macrophages might play an important role in lymph node metastasis. Higher counts of either macrophage type were associated with increased risk of lymph node metastasis, and the M2 macrophage count could potentially be a marker for evaluating prognosis.

We found the average survival time was $106.1 \pm 10.2$ months in the low CD68 expressing group, and the 5-year survival rate was $76 \%$ (16/21). In the high CD68 expressing group, the average survival time was $56.3 \pm 9.1$ months and the 5 -year survival rate was $42 \%$ (10/24). We analyzed the expression of CD68 and CD34-MVD, age, clinical stage, size of postoperative residual lesion, histological grade, postoperative radiotherapy, and pathological types by the Cox regression model. The results show that the degree of infiltration of CD68-TAMs, the CD34-MVD expression, and the histological grading were all potential prognostic factors of patients with laryngeal cancer.

One of the more interesting results of this study was that CD34-MVD expression was positively associated with the density of TAMs in a multivariate analysis model that included pathological features. However, the detailed mechanism of such pathological activities is not yet fully understood. Although a previous LSCC study focused on the function of TAMs in ovarian cancer [30], other investigators have reported similar findings in breast cancer [31]. However, Ohri et al. [32] reported that in non-small cell lung carcinoma patients, the macrophage density is markedly higher among patients that survived for extended periods than in those who did not survive. Based on these facts, we also suggest that CD34-MVD expression regulated by TAMs affects cancer cell invasion and progression in patients with LSCC. However, we did not find a specific number and type of TAMs in our specimens. That is, that although higher expression of TAMs was detected in LSCC at the edge of tumors, such findings are not characteristics generally associated with CD34MVD expression.

The positive expression rate of CD68 did not correlate with tumor location, degree of tumor differentiation, or clinical state, but it did correlate with lymph node metastasis. CD34-MVD expression and count was significantly higher in the LNM positive group than the LNM negative group. The high expression of CD34-MVD in LNM positive tumors is likely because the tumor has new, stronger blood vessels forming and increased metastatic activity and ability. Therefore, a further detailed investigation would be necessary to confirm this correlation in LSCC.

In conclusion, high expression of CD68-TAMs and CD34MVD are significantly correlated with pathological features such as lymph node metastasis and the presence of metastases in patients with LSCC. In addition, its expression is also associated with the expression of CD34-MVD. CD68-TAMs play important roles in the gain of malignant potential and malignant aggressiveness, including cancer cell invasion. We speculated that CD68-TAMs are a significant stimulator for CD34-MVD. Finally, the high expression of CD68-TAMs in laryngeal cancer tissue and its positive correlation with CD34-MVD suggests that both play an important role in promoting the metastasis and angiogenesis of laryngeal squamous carcinoma tissue. Their expression was positively correlated with the prognoses of laryngeal cancer patients and they have potent as important prognostic markers of laryngeal cancer.

\section{CONFLICT OF INTEREST}

No potential conflict of interest relevant to this article was reported.

\section{REFERENCES}

1. Zhou L, Yang K, Andl T, Wickett RR, Zhang Y. Perspective of targeting cancer-associated fibroblasts in melanoma. J Cancer. 2015 Jun;6(8):717-26.

2. Weinberg RA. Twisted epithelial-mesenchymal transition blocks senescence. Nat Cell Biol. 2008 Sep;10(9):1021-3.

3. Cavallo F, De Giovanni C, Nanni P, Forni G, Lollini PL. 2011: the immune hallmarks of cancer. Cancer Immunol Immunother. 2011 Mar;60(3):319-26.

4. Jang JY, Lee JK, Jeon YK, Kim CW. Exosome derived from epigallocatechin gallate treated breast cancer cells suppresses tumor growth by inhibiting tumor-associated macrophage infiltration and M2 polarization. BMC Cancer. 2013 Sep;13:421.

5. Duray A, Demoulin S, Hubert P, Delvenne P, Saussez S. Immune suppression in head and neck cancers: a review. Clin Dev Immunol. 2010;2010:701657.

6. Bostrom MM, Irjala H, Mirtti T, Taimen P, Kauko T, Algars A, et al. Tumor-Associated macrophages provide significant prognostic information in urothelial bladder cancer. PLoS One. 2015 Jul;10 (7):e0133552.

7. Li H, Zhang J, Chen SW, Liu LL, Li L, Gao F, et al. Cancer-associated fibroblasts provide a suitable microenvironment for tumor development and progression in oral tongue squamous cancer. J Transl Med. 2015 Jun;13:198.

8. Kim S, Cho SW, Min HS, Kim KM, Yeom GJ, Kim EY, et al. The expression of tumor-associated macrophages in papillary thyroid carcinoma. Endocrinol Metab (Seoul). 2013 Sep;28(3):192-8.

9. Pirila E, Vayrynen O, Sundquist E, Pakkila K, Nyberg P, Nurmennie$\mathrm{mi}$ S, et al. Macrophages modulate migration and invasion of human tongue squamous cell carcinoma. PLoS One. 2015 Mar;10(3): 
e0120895.

10. Boas DS, Takiya CM, Gurgel CA, Cabral MG, Santos JN.Tumor-infiltrating macrophage and microvessel density in oral squamous cell carcinoma. Braz Dent J. 2013;24(3):194-9.

11. Lin JY, Li XY,Tadashi N, Dong P. Clinical significance of tumor-associated macrophage infiltration in supraglottic laryngeal carcinoma. Chin J Cancer. 2011 Apr;30(4):280-6.

12. Klimp AH, Hollema H, Kempinga C, van der Zee AG, de Vries EG, Daemen T. Expression of cyclooxygenase-2 and inducible nitric oxide synthase in human ovarian tumors and tumor-associated macrophages. Cancer Res. 2001 Oct;61(19):7305-9.

13. Dineen SP, Lynn KD, Holloway SE, Miller AF, Sullivan JP, Shames DS, et al. Vascular endothelial growth factor receptor 2 mediates macrophage infiltration into orthotopic pancreatic tumors in mice. Cancer Res. 2008 Jun;68(11):4340-6.

14. Ding T, Xu J, Wang F, Shi M, Zhang Y, Li SP, et al. High tumor-infiltrating macrophage density predicts poor prognosis in patients with primary hepatocellular carcinoma after resection. Hum Pathol. 2009 Mar;40(3):381-9.

15. Green CE, Liu T, Montel V, Hsiao G, Lester RD, Subramaniam S, et al. Chemoattractant signaling between tumor cells and macrophages regulates cancer cell migration, metastasis and neovascularization. PLoS One. 2009 Aug;4(8):e6713.

16. Noy R, Pollard JW. Tumor-associated macrophages: from mechanisms to therapy. Immunity. 2014 Jul;41(1):49-61.

17. Kuribayashi K, Motoyama S, SasakiT, Sato Y, Yoshino K, Wakita A, et al. C-reactive protein reduces the relative number of tumor-associated M2 macrophages and intratumoral angiogenesis in mice. Tohoku J Exp Med. 2014;233(4):249-55.

18. Downey CM, Aghaei M, Schwendener RA, Jirik FR. DMXAA causes tumor site-specific vascular disruption in murine non-small cell lung cancer, and like the endogenous non-canonical cyclic dinucleotide STING agonist, 2'3'-cGAMP, induces M2 macrophage repolarization. PLoS One. 2014 Jun;9(6):e99988.

19. RiabovV, Gudima A,Wang N, Mickley A, Orekhov A, Kzhyshkowska J. Role of tumor associated macrophages in tumor angiogenesis and lymphangiogenesis. Front Physiol. 2014;5:75.

20. Zhang B, Zhang Y, Zhao J, Wang Z, Wu T, Ou W, et al. M2-polarized macrophages contribute to the decreased sensitivity of EGFR-TKIs treatment in patients with advanced lung adenocarcinoma. Med
Oncol. 2014 Aug;31(8):127.

21. Jung KY, Cho SW, Kim YA, Kim D, Oh BC, Park DJ, et al. Cancers with higher density of tumor-associated macrophages were associated with poor survival rates. J Pathol Transl Med. 2015 Jul;49(4):31824.

22. Guo C, Buranych A, Sarkar D, Fisher PB, Wang XY. The role of tumor-associated macrophages in tumor vascularization. Vasc Cell. 2013 Dec;5(1):20.

23. Baeriswyl V, Christofori G. The angiogenic switch in carcinogenesis. Semin Cancer Biol. 2009 Oct;19(5):329-37.

24. Lin EY, Pollard JW. Tumor-associated macrophages press the angiogenic switch in breast cancer. Cancer Res. 2007 Jun;67(11):5064-6.

25. Watari K, Shibata T, Kawahara A, Sata K, Nabeshima H, Shinoda A, et al. Tumor-derived interleukin-1 promotes lymphangiogenesis and lymph node metastasis through M2-type macrophages. PLoS One. 2014 Jun;9(6):e99568.

26. Bolat F, Kayaselcuk F, Nursal TZ, Yagmurdur MC, Bal N, Demirhan B. Microvessel density, VEGF expression, and tumor-associated macrophages in breast tumors: correlations with prognostic parameters. J Exp Clin Cancer Res. 2006 Sep;25(3):365-72.

27. Zhang M, He Y, Sun X, Li Q, Wang W, Zhao A, et al. A high M1/M2 ratio of tumor-associated macrophages is associated with extended survival in ovarian cancer patients. J Ovarian Res. 2014 Feb;7:19.

28. Lin CN, Wang CJ, Chao YJ, Lai MD, Shan YS. The significance of the co-existence of osteopontin and tumor-associated macrophages in gastric cancer progression. BMC Cancer. 2015 Mar;15:128.

29. Chen SJ, Zhang QB, Zeng LJ, Lian GD, Li JJ, Qian CC, et al. Distribution and clinical significance of tumour-associated macrophages in pancreatic ductal adenocarcinoma: a retrospective analysis in China. Curr Oncol. 2015 Feb;22(1):e11-9.

30. Colvin EK. Tumor-associated macrophages contribute to tumor progression in ovarian cancer. Front Oncol. 2014 Jun;4:137.

31. Deng R, Wang SM, Yin T, Ye TH, Shen GB, Li L, et al. Dimethyl sulfoxide suppresses mouse 4T1 breast cancer growth by modulating tumor-associated macrophage differentiation. J Breast Cancer. 2014 Mar;17(1):25-32.

32. Ohri CM, Shikotra A, Green RH, Waller DA, Bradding P. Macrophages within NSCLC tumour islets are predominantly of a cytotoxic M1 phenotype associated with extended survival. Eur Respir J. 2009 Jan;33(1):118-26. 